\title{
Cooling of a lattice granular fluid as an ordering process
}

\author{
A. Baldassarri ${ }^{1}$, U. Marini Bettolo Marconi ${ }^{1}$, A. Puglisi ${ }^{2}$ \\ ${ }^{1}$ INFM Udr Camerino, Univ. di Camerino, Dip. Matematica e Fisica, Via Madonna delle Carceri I-62032 Camerino, Italy \\ 2 Universitá "La Sapienza", P.le A. Moro 2, 00185 Roma, Italy
}

(October 28, 2018)

\begin{abstract}
We present a new microscopic model of granular medium to study the role of dynamical correlations and the onset of spatial order induced by the inelasticity of the interactions. In spite of its simplicity, it features several different aspects of the rich phenomenology observed in granular materials and allows to make contact with other topics of statistical mechanics such as diffusion processes, domain growth, persistence, aging phenomena. Interestingly, while local observables being controlled by the largest wavelength fluctuations seem to suggest a purely diffusive behavior, the formation of spatially extended structures and topological defects, such as vortices and shocks, reveals a more complex scenario.
\end{abstract}

Pacs: 5.40, 64.60.C, 45.70.M

The effort devoted during the last decades to investigate off equilibrium systems has achieved a series of successes by virtue of a combination of experiments, numerical simulations, exact theoretical results and clever phenomenological arguments, but our comprehension of the area is far from complete. Among these systems granular materials (GM), i.e. assemblies of macroscopic particles which dissipate their energy through inelastic collisions and frictional forces, have acquired a special rank due to their complex phenomenology often intriguing and only partly understood 1 .

The existing theoretical approaches are based either on realistic descriptions of the grains or on idealized models, which in virtue of their major simplicity lend themselves to analytic solutions or to efficient computations. Furthermore such an idealized modeling can deliberately sacrifice likeness to reality, in order to identify the physical mechanism responsible for the salient features of the system. In such a spirit, we shall discuss a minimal model based on the simplest rule which describes the inelasticity of collisions and local momentum conservation.

Years ago, S. Ulam showed that an ensemble of elastic particles, starting from an arbitrary configuration, converged to a Maxwell equilibrium distribution, postulating a simple redistribution law of the kinetic energies of randomly selected pairs to simulate the effect of binary collisions in an elastic gas [2]. Ben Naim and Kaprivski (BK), recently, performed a variation over this theme, by letting the particles endowed with a scalar velocity to dissipate inelastically a fraction of the relative kinetic energy at each collision and found that the total kinetic energy decreases exponentially with time [3]. Both models fulfill Boltzmann's molecular chaos hypothesis, and consequently rule out the formation of dynamical correlations. On the other hand computer simulations have shown the appearance of a shear instability, i.e. vortices, during the cooling process, before the spontaneous formation of density clusters. In an interesting series of papers, Ernst and collaborators have put forward a mesoscopic theory of these phenomena, making a connection with phase ordering kinetics $[6]$.

In the present work we shall introduce and study a new microscopic model which preserves the simplicity of the approach á la Ulam, and displays a complexity similar to that observed in granular systems. The focus of our study will be on the statistics of the velocity field and on its spatial and temporal correlations, stressing the analogies and the differences with related models aimed to describe off equilibrium systems.

We introduce our dynamical model by associating a d-dimensional velocity field $\mathbf{v}_{\mathbf{i}}$ with each node of a ddimensional lattice; at each time step a nearest neighbor pair $(i, j)$ is randomly selected and the two velocities are updated according to the rule:

$$
\begin{aligned}
& \mathbf{v}_{\mathbf{j}}^{\prime}=\mathbf{v}_{\mathbf{j}}+\Theta\left(-\left(\mathbf{v}_{\mathbf{i}}-\mathbf{v}_{\mathbf{j}}\right) \hat{\sigma}\right) \frac{1+\alpha}{2}\left(\left(\mathbf{v}_{\mathbf{i}}-\mathbf{v}_{\mathbf{j}}\right) \hat{\sigma}\right) \hat{\sigma} \\
& \mathbf{v}_{\mathbf{i}}^{\prime}=\mathbf{v}_{\mathbf{i}}-\Theta\left(-\left(\mathbf{v}_{\mathbf{i}}-\mathbf{v}_{\mathbf{j}}\right) \hat{\sigma}\right) \frac{1+\alpha}{2}\left(\left(\mathbf{v}_{\mathbf{i}}-\mathbf{v}_{\mathbf{j}}\right) \hat{\sigma}\right) \hat{\sigma}
\end{aligned}
$$

where $\hat{\sigma}$ represents the unit vector pointing from site $\mathbf{i}$ to $\mathbf{j}, \Theta$ is the Heaviside function which enforces the kinematic constraint and $\alpha$ the normal restitution parameter. We shall measure time in the non dimensional number of collisions per particle. In each elementary collision (see Eq.(1D) the total linear and angular momentum are conserved, whereas a fraction $\left(1-\alpha^{2}\right) / 4$ of the relative kinetic energy is dissipated. The inelasticity of the collisions has the effect of reducing the quantity $\left|\left(\mathbf{v}_{\mathbf{i}}-\mathbf{v}_{\mathbf{j}}\right) \hat{\sigma}\right|$, i.e. induce a partial allignement of the velocities. Hereafter we report the study performed in two dimensions on a triangular lattice [9].

The freely cooling process exhibits striking similarities with the quench from an initially stable disordered phase to a low temperature phase in a magnetic system: whereas in a standard quench process [10] one considers the process by which a thermodynamic system, brought out of equilibrium by a sudden change of an external constraint, such as temperature or pressure, finds its new 


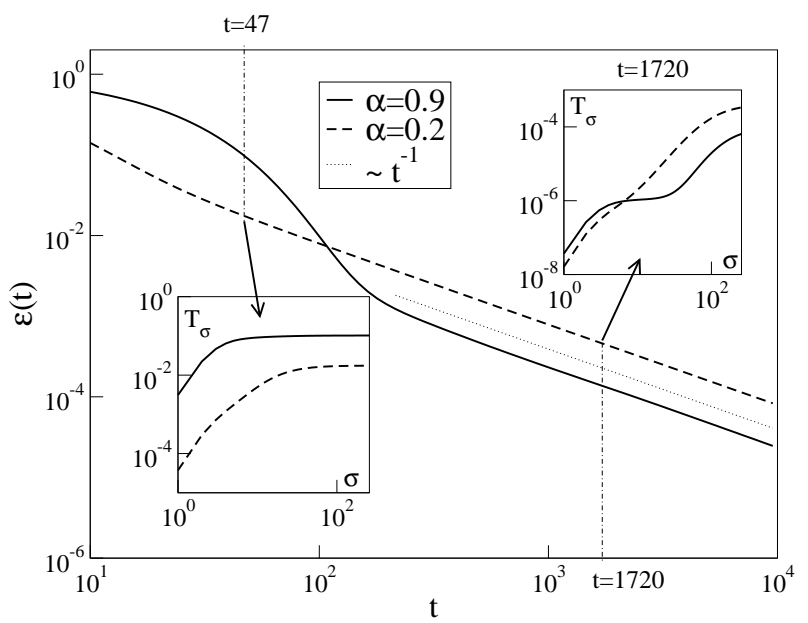

FIG. 1. Energy decay for $\alpha=0.9$ and $\alpha=0.2\left(1024^{2}\right.$ sites). The dotted line $\sim 1 / t$ is a guide to the eye for the asymptotic energy decay. In the insets we reported the scale dependent temperature, $T_{\sigma}$, defined in the text, as function of the coarse graining size $\sigma$ for $t=47$ and $t=1720$. The total energy per particle and $T_{\sigma}$ remain nearly indistinguishable in the early incoherent regime, but for $\sigma<L(t)$ the thermal energy becomes much smaller than the kinetic energy, a clear indication of the onset of macroscopic spatial order.

equilibrium state, in a GM one wants to study the relaxation of a fluidized state, after the external driving force (whose role is to reinject the energy dissipated by the collisions, keeping the system in a statistically steady state) is switched-off abruptly at some time $t=0$. The rotational symmetry of the order parameter $\mathbf{v}_{\mathbf{i}}$ and the momentum conserving interaction determine the presence of many configurations having comparable dissipation rates. Due to their competition the system does not relax immediately towards a motionless state, but displays a phenomenology similar to that observed in a coarsening process.

One sees from Fig.1 that during the initial stage, the total energy per particle $\epsilon(t)=\sum v_{i}(t)^{2} / N$ is dissipated at an exponential rate $\tau^{-1}=\left(1-\alpha^{2}\right) / 4$. This can be deduced from Eq.(11) imposing that each 'spin' fluctuates independently of the others. For times larger than $t \sim t_{c}$, of the order of $\tau$, the system displays a crossover to a different regime, where the cooperative effects become dominant and the average energy per particle decay as $\epsilon(t) \sim t^{-1}$. Such a behavior agrees with inelastic hard spheres simulations (IHSS) reported in [7]. As shown below, the crossover from one regime to the other is due to the formation of a macroscopic velocity field. This is analogous to the formation of magnetic domains in standard quench processes. After the formation stage these regions start to compete to homogenize, causing a conversion of kinetic energy into heat by viscous heating, i.e. act against the collisional cooling and lead to a slower decay of the energy [6].

Within the early regime the velocity distribution devi-

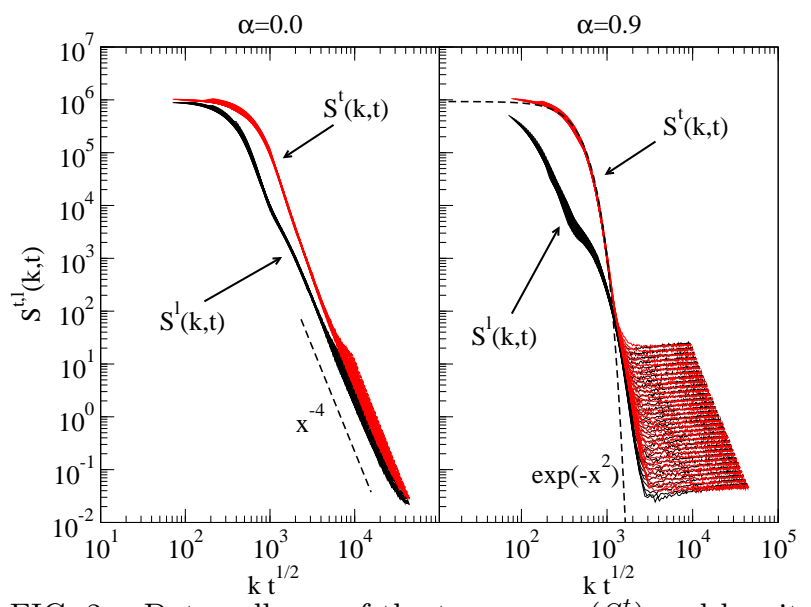

FIG. 2. Data collapse of the transverse $\left(S^{t}\right)$ and longitudinal $\left(S^{l}\right)$ structure functions for $\alpha=0$. and $\alpha=0.9$ (system size $1024^{2}$ sites, times ranging from $t=500$ to $\left.t=10^{4}\right)$. The wavenumber $k$ has been multiplied by $\sqrt{t}$. Notice the presence of the plateaux for the more elastic system. For comparison we have drawn the laws $x^{-4}$ and $\exp \left(-x^{2}\right)$.

ates sensibly from a Maxwell distribution (corresponding to the same average kinetic energy), but displays fatter tails, a phenomenon which mirrors the behavior of the BK [3] model. The existence of these tails seems to be due to the lack of spatial correlations, intrinsically absent at all times in their model, whereas negligible in ours up to $t_{c}$. When the energy begins to decay as $t^{-1}$ the velocity distribution turns Gaussian. Interestingly, the smaller the inelasticity, the faster is the energy dissipation, a phenomenon observed in IHSS [8].

The most relevant information about the spatial ordering process is contained in the equal-time structure functions, i.e. the Fourier transforms of the velocity correlation function:

$$
S^{t, l}(k, t)=\sum_{\hat{k}} \mathbf{v}^{\mathbf{t}, \mathbf{l}}(\mathbf{k}, t) \mathbf{v}^{\mathbf{t}, \mathbf{l}}(-\mathbf{k}, t)
$$

where the superscripts $t, l$ indicate the transverse and longitudinal components of the field with respect to the wave vector $\mathbf{k}$ and the sum $\sum_{\hat{k}}$ is over a circular shell of radius $k$. Such structure factors, if rewritten in terms of the variable $\left(k t^{1 / 2}\right)$ display fairly good data collapse, apart from the large $k$ region, and identify two growing lengths $L^{t, l}(t)$ (see Fig.21). Considering the sum rule $\epsilon(t)=\sum_{k}\left[S^{t}(k, t)+S^{l}(k, t)\right]$ we observed that in the early 'exponential' regime the contribution from the two terms is approximately equal, whereas for times larger than $t_{c}$ and $\alpha$ not too small most of the kinetic energy remains stored in the transverse field, while the longitudinal component decays faster, with an apparent exponent $t^{-2}$.

The findings concerning the energy decay, the distribution of the velocity field, and the growth of $L^{t, l}(t)$, lead to the conclusion, that, if the observation time is longer than the time between two collisions and if the 
spatial scale is larger than the lattice spacing, the system behaves as if its evolution were governed by a diffusive dynamics [6]. To be more precise, let us consider a vector field $\vec{\phi}(x, t)$ which evolves according to the law $\partial_{t} \vec{\phi}=\nu \nabla^{2} \vec{\phi}$ starting from a random uncorrelated initial condition. The explicit solution shows that $\vec{\phi}(x, t)$ is asymptotically Gaussian distributed, with a variance $<\vec{\phi}(x, t) \vec{\phi}(x, t)>\propto t^{-d / 2}$. The structure factors $S^{t, l}(k, t)$ assume a scaling form $S^{t, l}(k, t)=s(k L(t))$ where $L(t)=\sqrt{t}$. Furthermore, we compared the twotime autocorrelation $C\left(t_{1}, t_{2}\right)=\sum_{i} \mathbf{v}_{i}\left(t_{1}\right) \mathbf{v}_{i}\left(t_{2}\right) / N$ with $C_{\phi}\left(t_{1}, t_{2}\right)=<\phi\left(x, t_{1}\right) \phi\left(x, t_{2}\right)>$, whose expression reads:

$$
\frac{C_{\phi}\left(t_{1}, t_{2}\right)}{C_{\phi}\left(t_{1}, t_{1}\right)}=\frac{2}{\left(1+\frac{t_{1}}{t_{2}}\right)}
$$

During a short time transient, the autocorrelation function of our model differs from $C_{\phi}$, since it depends on $t_{1}-t_{2}$, i.e. it is time translational invariant $(T T I)$. Later, $C\left(t_{1}, t_{2}\right)$ reaches the "aging" regime and depends only on the ratio $x=t_{1} / t_{2}$. Something similar occurs in a coarsening process, where the autocorrelation of the local magnetization $a\left(t_{w}, t_{w}+\tau\right)$ reaches, for large $\tau$ (but $\tau<<t_{w}$ ), a constant value $m_{e q}^{2}(T)$, that is the square of the equilibrium magnetization. Obviously, for $T \rightarrow 0$, $m_{e q}^{2} \rightarrow 1$ and the TTI transient regime disappears. The short time transient in our model is analogous to such a TTI regime, with the difference that the cooling process imposes a decreasing temperature $T\left(t_{w}\right) \rightarrow 0$, that progressively erodes the TTI regime. The same dependence on the TTI manifests itself in the angular autocorrelation:

$$
A\left(t, t_{w}\right)=\frac{1}{N} \sum_{i} \cos \left(\theta_{i}\left(t+t_{w}\right)-\theta_{i}\left(t_{w}\right)\right) .
$$

Again, for large waiting times $t_{w}$ this function assume the diffusive $t / t_{w}$ scaling form, but for a small fixed $t_{w}$, displays a minimum and a small peak before decreasing at larger $t$ (see Fig.3). The non-monotonic behavior of $A\left(t, t_{w}\right)$ suggests that the initial direction of the velocity induces a change in the velocities of the surrounding particles, which in turn generates, through a sequence of correlated collisions, a kind of retarded field oriented as the initial velocity. As $t_{w}$ increases the maximum is less and less pronounced.

In spite of these first results, that seem to give support to the idea that the model dynamics is purely diffusive [11], the model is more complex. The main evidence stems from the following facts:

i) the structure functions do not have the typical Gaussian tails of a diffusive system, due to the non linearity represented by the kinematic factor in Eq.(11) and the shapes of $S^{t, l}(k, t)$ display three different regions: a longwavelength region which is diffusive in character; an intermediate region where the structure functions decay as

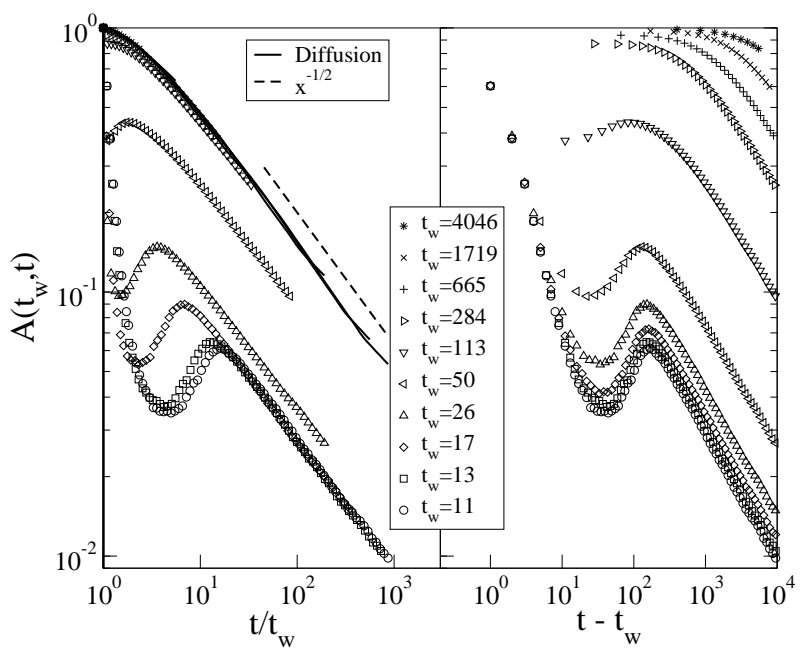

FIG. 3. Angular autocorrelation function $A\left(t, t_{w}\right)$ for different values of the waiting time $t_{w}$ and $\alpha=0.9$ (1024 ${ }^{2}$ sites). The graph on the left shows the convergence to the $t / t_{w}$ diffusive scaling regime, for large $t_{w}$. For small $t_{w}$, a local minimum is visible (for such a quasi elastic dynamics). In the graph on the right the same data are plotted vs $t-t_{w}$ : note that the small $t_{w}$ curves tend to collapse. For higher $t_{w}$ the position of the local minimum does not move sensibly, but its value grows and goes to 1 for large $t_{w}$.

$k^{-\beta}$ with $\beta \sim 4$; a plateau region where $S^{t, l}$ decay in time with a power law $t^{-2}$ but remain nearly constant with respect to $k$ (for $r>0$ );

ii) the Fourier modes interact and an initial shear state, obtained assuming the initial configuration to be a plane wave, decays into shorter wavelength modes by a mechanism of period doubling; that is to say, contrary to the diffusion, plane waves are not eigenmodes.

The existence of the quasi-elastic plateaux is the fingerprint of localized fluctuations which, for small inelasticity, propagate and are damped less than exponentially. A small $\alpha$ determines a rapid locking of the velocities of neighboring elements to a common value, while in the case of $\alpha \rightarrow 1$, short range small amplitude disorder persists within the domains, breaking simple scaling of $S^{t, l}$ for large $k$ and having the effect of a self induced noise.

One can characterize such internal noise by means of an average local granular temperature $T_{\sigma}$, i.e. a measure of the variance of $\mathbf{v}_{\mathbf{i}}$ with respect to the local average of $\mathbf{v}$ within a region of linear size $\sigma$. Obviously, since when $\sigma \rightarrow \infty$ the local average tends to the global (zero) momentum, then $T_{\sigma} \rightarrow \epsilon$, as shown in the insets of Fig.1. For $\sigma<L(t)$, instead, $T_{\sigma}<\epsilon$. For quasi elastic systems $T_{\sigma}$ exhibits a plateau for $1 \ll \sigma \ll L(t)$ that identify the strength of the internal noise. The local temperature ceases to be well defined for smaller $\alpha$ due to the absence of scale separation between microscopic and macroscopic fluctuations in the strongly inelastic regime [8]. The existence of a $L^{-2}(t) k^{-4}$ region in the structure functions is consistent with Porod's law 10 and is 


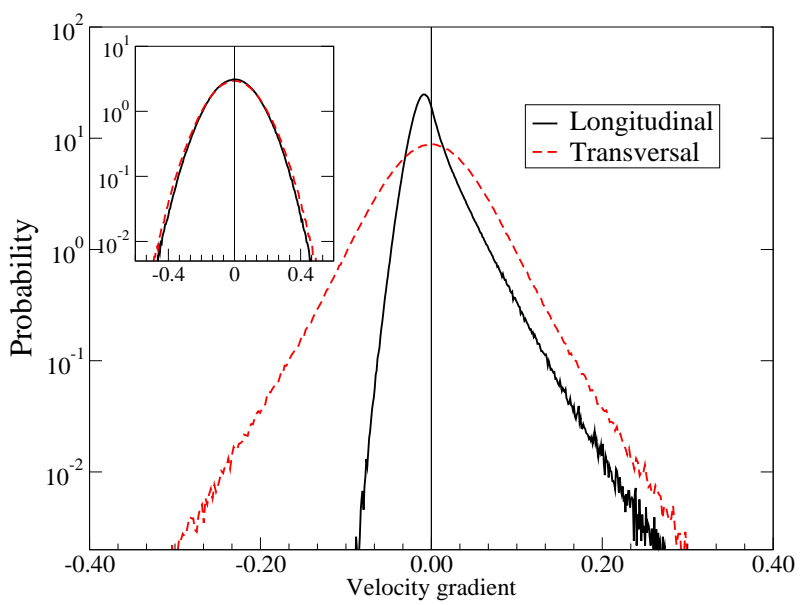

FIG. 4. Probability densities of the longitudinal and transverse velocity increments. The main figure shows the p.d.f. of the velocity gradients $(R=1)$. The inset shows the Gaussian shape measured for $R=40$ (larger than $L(t)$ for this simulation: $\alpha=0.2, t=620$, system size $\left.2048^{2}\right)$.

the signature of the presence of vortices, a salient feature of the cooling process. Vortices form spontaneously and represent the boundaries between regions which selected different orientations of the velocities during the quench and are an unavoidable consequence of the conservation laws which forbid the formation of a single domain. With the random initial conditions adopted, vortices are born at the smallest scales and subsequently grow in size by pair annihilation, conserving the total charge. By locating the vortex cores, we measured the vortex density $\rho_{v}(t)$, which represents an independent measure of the domain growth, and in fact it decays asymptotically $\left(t \gg t_{c}\right)$ as an inverse power of time, i.e. $L_{v}(t)=\rho_{v}^{-1 / 2} \propto t^{1 / 2}$. The vortex distribution turns out to be not uniform for $\alpha$ not too small. Its inhomogeneity is characterized by the correlation dimension $d_{2}$ : $H(R)=N_{v}^{-2} \sum_{i<j} \Theta\left(R-\left(\mathbf{r}_{\mathbf{i}}-\mathbf{r}_{\mathbf{j}}\right)\right) \sim R^{d_{2}}$ where the $r_{i}$ are the core locations. For $\alpha \rightarrow 1$ the vortices are clusterized $\left(d_{2}<2\right)$ i.e. do not fill homogeneously the space, whereas at smaller $\alpha$ their distribution is homogeneous $\left(d_{2} \rightarrow 2\right)$.

Vortices are not the only topological defects of the velocity fields. In fact we observe shocks, similarly to recent experiments in rapid granular flows [13]. Shocks have a major influence on the statistics of velocity field, i.e. on the probability distributions of the velocity increments. The probability density function (p.d.f.) of the longitudinal increment

$$
\Delta_{l}(\mathbf{R})=\frac{1}{N} \sum_{\mathbf{i}}\left(\mathbf{v}_{\mathbf{i}+\mathbf{R}}-\mathbf{v}_{\mathbf{i}}\right) \cdot \frac{\mathbf{R}}{R}
$$

is shown in Fig 4 for $R=1$ (longitudinal velocity gradient) in the main frame, and for $R=40>L(t)$ in the inset. For small $R \ll L(t)$ the longitudinal increment p.d.f. is skewed with an important positive tail, whereas for $R \gg L(t)$ it turns Gaussian. The distribution of transverse increments $\left(\mathbf{v}_{\mathbf{i}+\mathbf{R}}-\mathbf{v}_{\mathbf{i}}\right) \times \hat{\mathbf{R}}$, instead, is always symmetric, but non Gaussian distributed for small $R$. A similar situation exists in fully developed turbulence [12].

To conclude, our model provides a link between the microscopic rules of granular dynamics and its hydrodynamical description. It allows to follow the cooling of a granular material and the build up of velocity correlations, by means of efficient numerical measures of structure factors, two-time correlations and topological defects. The data analysis reveals the presence of vortices, shocks and internal noise and suggests the existence of a scale separation only in the case of quasi elastic systems, which is instead suppressed for large inelasticities.

Even independently from the problem of granular flows, the model represents a simple but unusual phase ordering system. In fact, despite the apparently purely diffusive aspects shown by one-point quantities, it displays anomalous statistics of spatial properties for the order parameter field as witnessed by the velocity gradient p.d.f. and by the structure functions.

Acknowledgments. We thank E. Caglioti, M. Cencini, L.F. Rull, A. Vulpiani and S. Zapperi for several useful discussions.

[1] H.M. Jaeger, S.R. Nagel and R.P. Behringer, Rev.Mod.Phys. 68, 1259 (1996); T. Pöschel, S. Luding (Eds.) Granular Gases, Springer, Berlin (2001).

[2] S. Ulam, Adv.Appl.Math. I, 7-21 (1980).

[3] E. Ben-Naim, P.L. Krapivsky, Phys.Rev. E, 61 R5 (2000).

[4] N. Sela and I. Goldhirsh, Phys.Fluids 7, 507 (1995).

[5] E. Ben-Naim, S.Y. Chen, G.D. Doolen, and S. Redner, Phys.Rev.Lett. 83 4069-4072 (1999).

[6] T.P.C. van Noje, M.E. Ernst, R. Brito and J.A.G. Orza, Phys.Rev.Lett. 79, 411 (1997).

[7] J.A.G. Orza, R. Brito, M.H. Ernst, cond-mat/002383.

[8] S.McNamara, Phys.Fluids A 5, 3056 (1993).

[9] The 1-d version of our model compares well with models of hard rods (see [5]), since in both cases each particle collides only with its neighbors (A.Baldassarri, U. Marini Bettolo and A.Puglisi, in preparation).

[10] A.J. Bray, Adv.Phys. 43 , 357 (1994).

[11] Even the persistence exponent $\theta$, defined through the decay $N_{s} \sim t^{-\theta}$ of the number of sites where a velocity component never changed its sign up to time $t$, agrees with the analogous exponent of the diffusion equation (we measured $\theta=0.18$ ).

[12] R. Benzi, L. Biferale, G. Paladin, A. Vulpiani, and M. Vergassola, Phys.Rev.Lett. 67, 2299 (1991).

[13] E. Rericha et al. cond-mat/0104474.

[14] S. Aumaitre, S. Fauve, S. McNamara and P. Poggi, Eur.Phys.J. B 19, 449 (2001). 